\title{
A systematic review of statistical process control implementation in the food manufacturing industry
}

\begin{abstract}
This paper is a systematic review of the literature on statistical process control (SPC) implementation in the food industry. Using systematic searches across three decades of publications, 41 journal articles were selected for the review. Key findings of the review include motivations: to reduce product defects and to follow the food law and regulations (benefits); barriers: high resistance to change and lack of sufficient statistical knowledge; and (limitations) an absence of statistical thinking and a dearth of SPC implementation guidelines. Further findings highlight the predominance of publications from the USA and the UK within this topic. Future research directions concerning SPC implementation issues as well as a ready reference of the SPC literature in the food manufacturing industry are also discussed.
\end{abstract}

Keyword: Statistical process control; Food manufacturing industry; Systematic review; Continuous improvement; Statistical thinking 